\title{
Enfoques pluri-inter-transdisciplinarios en la prospectiva de desarrollo universitario: Un estudio de casos
}

Pluri-inter-transdisciplinary approaches in prospective university development:

A case study

Enfoques pluri-inter-transdisciplinares na perspectiva do desenvolvimento universitário: Um estudo de caso

\author{
Nicolás Díaz Barrera, ${ }^{\text {a }}$ Alejandra Soler Urzúa, ${ }^{\text {b }}$ Iván Oliva Figueroa ${ }^{\mathrm{c}}$ \\ ${ }^{\mathrm{a}}$ Universidad Austral de Chile, Programa de Doctorado en Ciencias Humanas. Valdivia, Chile. \\ Correo electrónico: ndiaz.lenguaje@gmail.com \\ ${ }^{\mathrm{b}}$ Correo electrónico: asoleru@gmail.com \\ ${ }^{\mathrm{c}}$ Universidad Austral de Chile, Facultad de Filosofía y Humanidades, Instituto de Filosofía y Estudios Edu- \\ cacionales. Valdivia, Chile. Correo electrónico: ivanoliva@uach.cl
}

\begin{abstract}
RESUMEN
El presente reporte de investigación desarrolla un marco teórico de referencia asociado a las nociones de complejidad y organización disciplinaria, en el contexto del entendimiento de los procesos de generación, comunicación, organización y legitimación del conocimiento en universidades chilenas. Desde esta base, se exponen y discuten los resultados de una investigación en torno a la interpretación de las bases epistemológicas subyacentes a las políticas y prospectivas de desarrollo de universidades chilenas en este contexto.
\end{abstract}

Palabras clave: interdisciplina, transdiciplina y universidad.

\begin{abstract}
This research paper develops a theoretical framework of reference associated with the notions of complexity and disciplinary organization in a context of understanding the creation, communication, organization and legitimization of knowledge in Chilean universities. From this base, this paper presents and discusses the results of an investigation regarding the interpretation of the epistemological bases underlying politics and prospective developmental policies in Chilean universities within this context.
\end{abstract}

Key words: interdiscipline, transdiscipline, university.

\section{RESUMO}

Este relatório de pesquisa desenvolve um marco teórico de referência associado às noções de complexidade e organização disciplinar no contexto do entendimento dos processos de geração, comunicação, organização e legitimação do conhecimento em universidades chilenas. A partir de essa base, são expostos e discutidos os resultados de uma investigação acerca da interpretação das bases epistemológicas subjacentes às políticas e perspectivas de desenvolvimento de universidades chilenas inseridas em tal contexto.

Palavras chave: Interdiscipline, transdiscipline, universidade.

* Trabajo de investigación enmarcado en el proyecto FONDECYT-INICIACIÓN 11080193 "Conocimiento, Complejidad y Transdisciplina: una investigación paradigmatológica en universidades chilenas”. 


\section{INTRODUCCIÓN}

Tomando en consideración las sucesivas crisis que se han presentado en la educación a nivel general y con énfasis en la educación terciaria, cabe preguntarse cuáles son las implicancias de contar con bases epistémicas críticas para otorgar una respuesta educativa consciente y acorde a los requerimientos sociales, develando los puntos de encuentro a las complejidades de la formación educativa como un fin en sí mismo y no como una herramienta, sin dejar de lado los estándares que se establecen a nivel mundial a favor del desarrollo propuesto por los países primer-mundistas.

En este contexto el informe emitido por la CNIC (Consejo Nacional de Innovación para la Competitividad, 2009) respecto al estudio realizado por la OECD y el Banco Mundial, la necesidad de revisar la carga académica de los programas de educación terciaria en las instituciones de educación superior chilenas, teniendo en cuenta tanto las necesidades nacionales como los estándares internacionales, con el fin de desarrollar programas académicos menos recargados y más eficientes en cuanto a la adquisición de conocimientos en base a competencias requeridas para el desenvolvimiento en la sociedad actual; así como también establecer una mayor articulación con los demás niveles de educación para reducir la brecha de conocimiento relevante necesario para el ingreso a la educación terciaria.

En este escenario, el estudio y conclusiones del Informe de la OECD (2008) han señalado que la pedagogía e investigación de la educación terciaria debe fomentar la relación de áreas diversas del conocimiento, en base a programas que asumen una mirada transdisciplinaria más extensa y formas más complejas de crear conocimiento. Dichos desafíos involucran una redefinición del rol de las universidades y la educación terciaria en general como, asimismo, esquemas de identidad disciplinar y las dinámicas relacionales de sus participantes.

El presente estudio trabaja sobre las bases de la vinculación disciplinar (multi-pluriinter-transdisciplina) y el paradigma del pensamiento complejo elaborado por el sociólogo Edgar Morin (2001, 2006a, 2006b). La investigación sitúa su planteamiento en relación a la convergencia de éstas propuestas en el contexto de la educación terciaria chilena, posicionando la investigación en tres establecimientos universitarios pertenecientes al Consejo de Rectores de Universidades Chilenas. Con una metodología de análisis hermenéuticodocumental desde el paradigma cualitativo, fue observada una tendencia transversal, con diversos grados de profundidad, hacia la apertura de las áreas del conocimiento desde las prospectivas políticas declaradas por estas instituciones. Así mismo, se visualiza, que de las tres áreas investigadas (Docencia, Investigación y Extensión) el Área de Docencia es el espacio de mayor tendencia hacia prospectivas de vinculación disciplinar y demuestra apertura hacia la integración de elementos de la teoría del pensamiento complejo dentro del proceso de enseñanza-aprendizaje. A su vez, fue posible observar focos específicamente transdisciplinarios en sus lineamientos, intencionando espacios socio-comunicativos, proyección y objetivos en las universidades investigadas.

En síntesis, el objetivo general de investigación fue cartografiar los elementos interpretativos asociados en las nociones de complejidad y vinculación disciplinar, presentes en la prospectiva política y estratégica de tres universidad del Consejo de Rectores de Universidades de Chile. 


\section{MARCO TEÓRICO REFERENCIAL}

Los cambios producidos en un mundo concebido por nuevos paradigmas socioculturales, económicos y políticos han levantado perspectivas epistemológicas acordes al derrumbe de fronteras informacionales generadas por el proceso de globalización iniciado en las últimas décadas al alero de la conectividad y la simultaneidad. Estos han generado un entrelazamiento y una vinculación importante entre el saber científico, las tecnologías y la vida cotidiana, transformando a escala mundial la vida de los seres humanos involucrados en los procesos de desarrollo transversal a todo nivel de estructura social. Estos cambios en Chile han sido radicales debido al avance económico realizado en las últimas décadas, de modo que no ha estado al margen de las vanguardias tecnológicas de la industria y la comunicación. Las personas han experimentado las ventajas y desventajas de estos avances, así como también de las nuevas formas en que el conocimiento se genera a partir de contextos muy diferentes de las décadas anteriores, principalmente por las transformaciones en el sistema económico de clausura a uno de apertura (Eyzaguirre, Marcel, Rodríguez y Tokman; 2005). A la vez, se ha situado al desarrollo como punto neurálgico de las políticas económicas y sociales implementadas en el país, pero levantando la problemática expresada desde amplios frentes sociales respecto a cuál es la naturaleza de éste desarrollo y hacia dónde se dirige.

Las nuevas formas de concebir este conocimiento generado desde un contexto social planetario en constante cambio, emergen paralelamente a estilos inéditos de producción industrial y organización política, en circunstancias de creciente complejidad social (Aronson, 2003) cuyas problemáticas requieren de consensos que superen las barreras disciplinares y sociales, con una multiplicidad de enfoques capaces de vislumbrar las necesidades globales que des-eliticen las soluciones planteadas para dichas interrogantes. De esta manera, las nuevas formas del conocer se han centrado en la participación del ser humano como punto clave de la resolución de conflictos de toda índole, dando lugar a la generación de incertidumbres como espacio neurálgico de nuevas oportunidades y no de barreras que desestructuren los avances realizados, de la mano con la creatividad como instrumento base de síntesis epistémica que no puede ser encasillada de forma uni-monodisciplinar. De esta manera, tomando la universidad como un espacio en el cual se presentan distintas matrices disciplinares que aparentemente descansan en medidas de especialización y no de interrelaciones, ni confluencia trans-interdisciplinar, se ubica como uno de los elementos constitutivos de generación de conocimiento y responsable de la creación de aperturas epistemológicas preexistentes. Gandarilla (2010) amplía la responsabilidad de la universidad más allá de planos epistemológicos y menciona que estas también tienen la labor de guiar a la sociedad como entes organizadores, capaces de abrir alternativas de elección y gestión paralelas a la política imperante constituyéndose como un espacio que se interrelaciona y retroalimenta con la sociedad.

Sotolongo y Delgado (2006) caracterizan el canon metodológico preponderante de los siglos XVIII y XIX dentro de un estricto saber racional que deslegitima los mitos y leyendas que no concuerdan con el método científico imperante; una permanente estrategia de fragmentación de la realidad física y cultural que origina disciplinas, especialidades y sub-especialidades incapaces de percibir la complejidad de lo real, dificultando la comprensión e interpretación de las problemáticas generadas en espacios más allá de los institucionales. De manera contextual, las bases paradigmáticas de la educación superior 
tradicional siguen impulsando esquemas cognitivos disyuntores que buscan las comprensión y el aprendizaje mediante la separación de las partes, mediante saberes inconexos que no dan espacios para la reflexión, la creación, el disentimiento, la curiosidad y la duda (Pereira en Oliva, 2008). Los distintos niveles educativos y de formación profesional se encuentran obsoletos a causa de los profundos cambios científicos y tecnológicos, y por los cambios emergentes en el orden social, económico y cultural. Las organizaciones educativas suman a su despiste actual, un creciente aislamiento institucional con respecto a los desafíos sociales y laborales en particular, y a las transformaciones de la cultura y la vida en general (Motta, 2002). Aronson (2003) sostiene que la historia de las instituciones de educación superior muestra la repetición de un patrón de respuestas que, tanto en el pasado como en el presente, impide su renovación. También manifiesta la postura respecto a la educación terciaria que describe la fragmentación del conocimiento actual. Las universidades han crecido desmedidamente, aprovechando cada oportunidad para involucrarse en nuevas actividades.

En un confuso proceso de desarrollo, los estudiantes se han convertido en cifras, aislados en sus áreas y en facultades cada vez menos interactivas y alejadas de lo que fue la comunidad sinérgica de la Academia de Platón. Esta postura nos indica la presencia de paradigmas económicos rigiendo los estatutos del sistema educativo terciario, demostrando que la universidad desde la postura actual es tratada como una empresa que obedece a funciones productivas que se alejan peligrosamente de las orientaciones educativas, formadoras de competencias teóricas y generadoras de conocimiento, como se establece en sus orígenes (Soler, 2010). Esto significa que los contextos de la formación inicial universitaria recrean las disciplinas de origen en sus modos de actuación (Corral \& Bar, 2012). Si bien estas recreaciones asumen formas en algunos aspectos próximas a la disciplina en cuestión, en otros se distancian atendiendo a las regulaciones de su propio contexto, ninguno demasiado situado en la praxis de sus conocimientos ni en su articulación con otros saberes.

Se entenderá por disciplina una categoría organizadora dentro del conocimiento científico, instituyendo en éste la división y la especialización disciplinar. Por más que se inserta en un contexto científico más amplio, una disciplina tiende a la autonomía por medio de la delimitación de sus fronteras a nivel técnico, metodológico y epistemológico (Morin, 2001a) más aún, está en el seno del conocimiento organizacional, que nace con una visión demarcada, dividida y tendiente a la especialización del trabajo respondiendo al paradigma dominante. Al tener una noción de disciplina como fenómeno particular, podemos indagar en nociones como pluridisciplina, multidisciplina, interdisciplina y transdisciplina. "los prefijos pluri y multi refieren a aspectos cuantitativos es decir varias o muchas disciplinas. En cambio los prefijos inter y trans aluden a relaciones recíprocas, actividades de cooperación, interdependencia e interpenetración" (Motta, 2002: 3). Gandarilla (2010) establece los elementos de objetividad, imparcialidad y neutralidad como criterios del rigor científico, cuya analogía estaría ligada al concepto de saber racional planteado por Sotolongo y Delgado (2006). En la medida que se establece como un conocimiento relevante y pertinente mediante validaciones académicas, generando la monopolización del conocimiento, sesgando las posibilidades de meta configuraciones extrapoladas del núcleo intelectual-universitario, la transdisciplina se ubicaría como opción resolutiva de estas problemáticas basadas en la incomunicación y parcelación de los saberes (Browne, 2006).

Caravantes García (citado en Oliva, 2008: 234) aludiendo a la transdisciplina menciona que "las disciplinas abren sus fronteras, pero pierden individualidad, aunque es 
posible definir transdisciplinariedad sobre la base de una conexión entre disciplinas sin pérdida de autonomía. Así el prefijo trans no denota pérdida de identidad, sino más bien relación; una condición inmanente a la reunión de disciplinas autorreferenciales, estrechamente conectadas y dependientes entre sî’. Max-Neff (2004), por su parte, considera que la interdisciplina se desarrolla en diversos niveles que comprenden cierta jerarquía de dominio, aludiendo a un esquema piramidal que sostiene las bases de ésta conceptualización. Los niveles jerárquicos se manifiestan en uno de corte empírico (como base), pragmático, ejemplificados en disciplinas específicas. Un nivel normativo, orientado a la planificación y diseño de sistemas. Por último, una cúspide valorativa que denota un nivel de superioridad ético-valórica, en donde están presentes principios filosóficos, teológicos y morales. Cambursano (2006) a su vez establece el trabajo transdisciplinario como un grupo interdisciplinario en el seno del cual los profesionales que lo conforman han re significado de su rol por efecto de la acción interactiva; de este modo llegan a trascender su propio espacio disciplinar. La disciplina, el equipo de trabajo, se deslizan del lugar central que pasa a ser ocupado por el tema o problema que reclama el abordaje específico.

De las diferentes visiones posibles de analizar respecto a la noción de complejidad, la de Edgar Morin (2001a) configura los elementos que permiten generar una conceptualización más allá de sistemas indivisibles, visualizando la recursividad, el caos y la incertidumbre como algo inherente al pensamiento, permitiendo establecer la complejidad como un sistema de redes de pensamientos y acciones imposibles de explicar con una articulación cartesiana de la realidad. Así, causa-efecto pierden sentido, porque aparece un correlato infinito en la configuración de la realidad, aludiendo a las espirales concéntricas que propone Morin ligado a la Teoría de Sistemas. Cada nivel de realidad es creado por situaciones anteriores sin la yuxtaposición que presupone un paradigma linealista. Según Wagensberg (en Gandarilla, 2010: 139) "el conocimiento de la complejidad interactúa entre dos nudos problemáticos: la cuestión del cambio (lo que hace referencia a la estabilidad y la evolución) y la relación entre los todos y sus partes (lo que hace referencia al problema de la estructura y la función)". Quedan implícitos en esta interacción los criterios que emergen a partir de la interretroacción de los fenómenos de distintas dimensiones que se conciben como móviles (sistemas no-estáticos, no-lineales) dentro de ciertos espacios de equilibrio (evolución-transformación, estabilidad-continuidad) y otra de estructuras fragmentadas desde un todo, que obedece a una condición de uso y pragmatismo.

Es necesario hablar de conocimientos y de realidades que se presentan desde la multiplicidad de enfoques complejizados, la diversidad de aprendizajes y saberes que nos llevan a una diversidad de perspectivas desde las cuales percibimos el mundo, más allá de una concepción acumulativa de saberes que han puesto al conocimiento dentro de variables medibles cuantitativamente. Toledo (2007) nos ayuda a comprender en un primer nivel que los sujetos que comparten un mismo acervo social de conocimientos y experiencias son provistos, por medio de su familiarización con los hábitos compartidos, de una competencia interpretativa elemental a partir de lo cual, escapando del núcleo autorreferencial del ser humano hacia su propio actuar hace posible comprender las rutinas de interacción de los otros, sus motivos y causas, en virtud de lo cual los meros individuos se convierten en auténticos actores sociales.

Esta aseveración de Toledo proporciona dos visiones que son fundamentales en la comprensión de la complejidad como posicionamiento epistemológico, diversificando nuestros esquemas de acción interna, desde la cual configuramos en el mundo y nos 
hacemos partícipes de él, hacia nuestra relación con otros, desde la cual configuramos aún mayores posibilidades que vuelven a actuar en nuestros esquemas de acción interna. Ligando esta descripción hacia el concepto de alteridad, descubrimos que las posibilidades del conocer se amplían a través del entramado social en vez de clausurarse y que las realidades se multiplican y se expanden hacia otras realidades que emergen (o se distinguen) desde estas configuraciones.

\section{METODOLOGÍA}

El posicionamiento de este trabajo se ubica en torno a una concepción sistémica, compleja y no-lineal para la construcción del conocimiento. Nuestro estudio es un trabajo de profundo diálogo entre elementos teóricos propios del marco referencial con elementos de integración contextual presentes de forma explícita e implícita dentro de las fuentes a analizar, siendo éstas últimas prospectivas políticas de tres establecimientos universitarios.

Se utilizó la hermenéutica como herramienta de análisis para observar fenómenos globales de significación, reiteración y asociación que quedan yuxtapuestos en la observación de sus elementos aislados y particulares. Mediante esto, se posibilitó la emergencia de dominios de apertura y clausura epistemológica-disciplinar, y la asociación de códigos invisibles en otro contexto. A partir de este análisis fue posible construir inductivamente una planilla de códigos (Figura 1) desde la cual se establecieron parámetros comparativos de frecuencia semiótica y la posterior discusión de resultados de los contextos universitarios investigados.

Schleiermacher, estudiado por Echeverría (1998), reflexiona sobre la hermenéutica como el arte del entendimiento a partir del diálogo. En el diálogo intervienen distintas visiones y perspectivas sobre la realidad, de forma que el lector aplica sus propios saberes a la interpretación de un texto. Esto trae consigo la incorporación de aspectos internos del sujeto para un mejor análisis; así, para acercarnos más fielmente a su intención, deben ser considerados los elementos pertenecientes a la dimensión valorativa del sujeto (Cárcamo, 2005).

En palabras de Beuchot (1997), el acto de interpretar es el de contextualizar o por lo menos es una parte y aspecto muy importante de ese acto, pues la comprensión es el resultado inmediato y hasta simultáneo de la contextualización. Interpretar es, a la vez, contextualizar y comprender un texto en el contexto.

Desde esta base, se procedió a un proceso de codificación que complementó estrategias de carácter inductivo y deductivo, en la sistematización de las diversas regularidades cualitativas observadas en los documentos. Para ello se generó deductivamente una matriz de códigos desde las principales bases teóricas asociadas a las implicancias epistemológicas de la noción de complejidad y organización disciplinar en la prospectiva universitaria, atendiendo inductivamente a la naturaleza específica de los documentos interpretados. 
Figura 1. Tabla de orden deductiva-inductiva que muestra los códigos y descriptores aplicados en la investigación

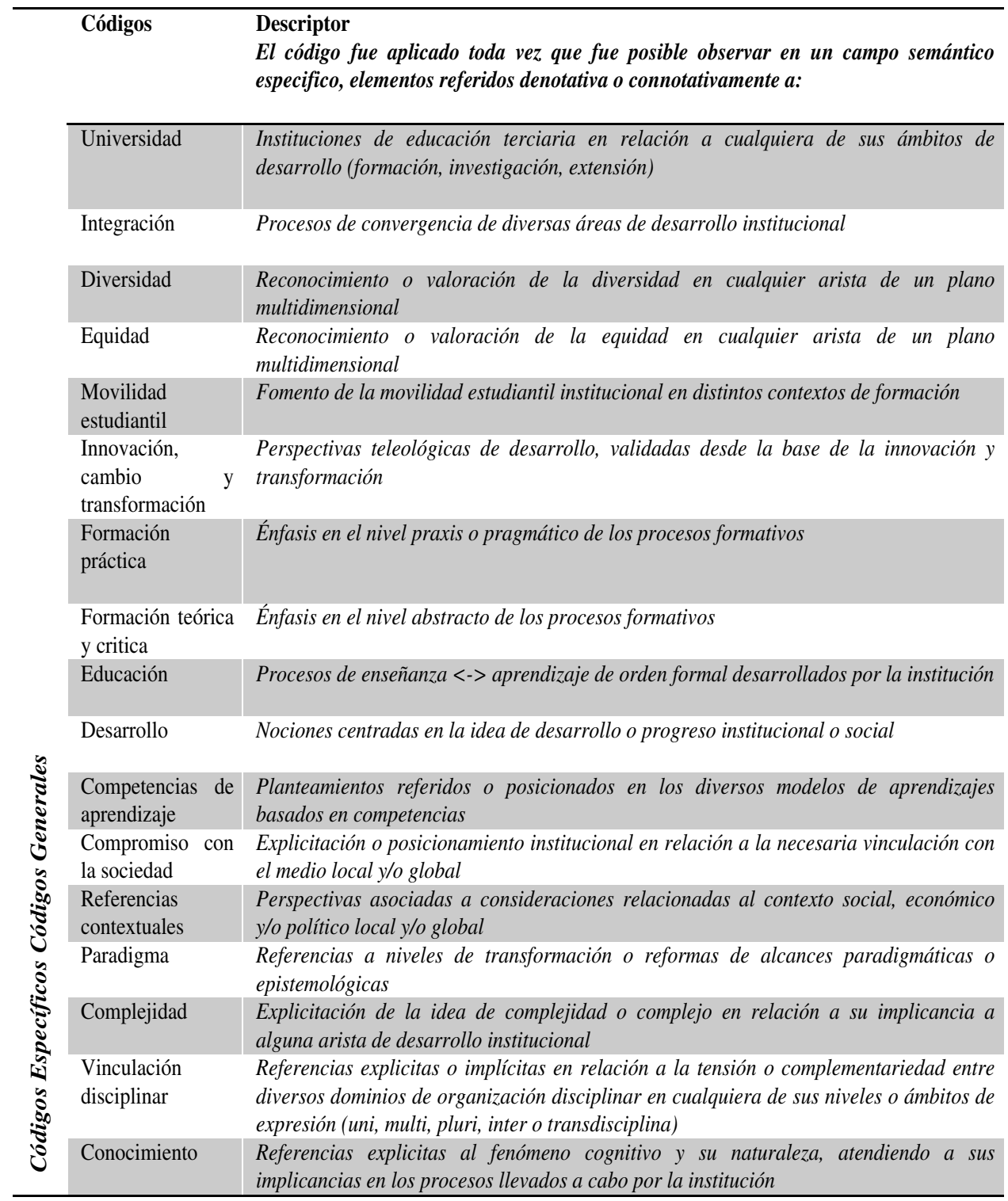

\section{CRITERIOS DE INCLUSIÓN Y FUENTES DOCUMENTALES}

Las tres universidades seleccionadas para esta investigación cumplieron con tres requisitos formales; a) pertenecer al Consejo de Rectores de Universidades Chilenas, b) definirse como universidades que desarrollan docencia, investigación y extensión, y c) cultivar al menos cuatro áreas disciplinares del conocimiento, según criterios OCDE. 
Para desarrollar el análisis hermenéutico, se utilizaron como fuentes primarias de trabajo documentos oficiales de las tres universidades escogidas en distintos niveles y dimensiones, buscando levantar desde ellos una interpretación cualitativa de su funcionamiento. Los niveles y dimensiones determinados para el análisis pretenden abarcar un universo amplio de aspectos que puedan dar evidencias de la postura rectora que toman las universidades. De este modo, existe un nivel macro (universidad) y micro (facultades). En cuanto a las dimensiones a trabajar, están referidas a docencia, investigación y extensión. Las fuentes utilizadas fueron las siguientes:

$i$. Documentos sobre políticas institucionales. Estos entregan, normalmente, orientación hacia la misión y visión a la institución, determinando la orientación sociopolítica y de mercado social que asume.

ii. Informes sobre autoevaluación Institucional. Entendidos como una evidencia de los procesos de gestión que desarrolla cada Universidad para analizar sus fortalezas, debilidades, oportunidades y amenazas, que sirvan de eje rector de las potencialidades que poseen y los elementos en los que debe enfatizarse la intervención a nivel institucional.

iii.Documentos de orientación curricular y planes estratégicos. Planes de las diversas facultades que den orientaciones en las diversas áreas de tratamiento.

iv. Políticas de extensión o vinculación con el medio.

Por otro lado, los documentos se clasifican por áreas que permiten un ordenamiento de acuerdo a la naturaleza de estos, las cuales fueron utilizadas como marco de indagación en esta investigación, a saber, áreas de investigación, docencia, extensión y mixtas.

\section{RESULTADOS}

Las siguientes gráficas radiales nos muestran las tendencias de la documentación estudiada en relación a los códigos de indagación y la presencia junto al espacio de movilidad en el cual convergen la totalidad de códigos. De esta manera, podemos observar de manera global que la situación de Caso 1 (Figura 2) presenta altos grados de afluencia en los códigos competencia de aprendizaje, desarrollo y referencias contextuales. Mientras tanto en el caso 2 (Figura 3) vinculación, desarrollo y formación son los códigos de mayor iteración. El Caso 3 (Figura 4) los códigos de mayor afluencia son competencias de aprendizaje, desarrollo y formación.

La complementaridad metodológica de los análisis de frecuencia e interacción de códigos supone que no solo es relevante la diversidad y frecuencia de codificación, sino también los efectos de interacción y contextualización de estos elementos de sentido en redes complejas de interpretación y prospectiva. Dicha complementariedad es desarrollada en el siguiente apartado del trabajo. 
Figura 2. Distribución de frecuencia de codificación Caso 1

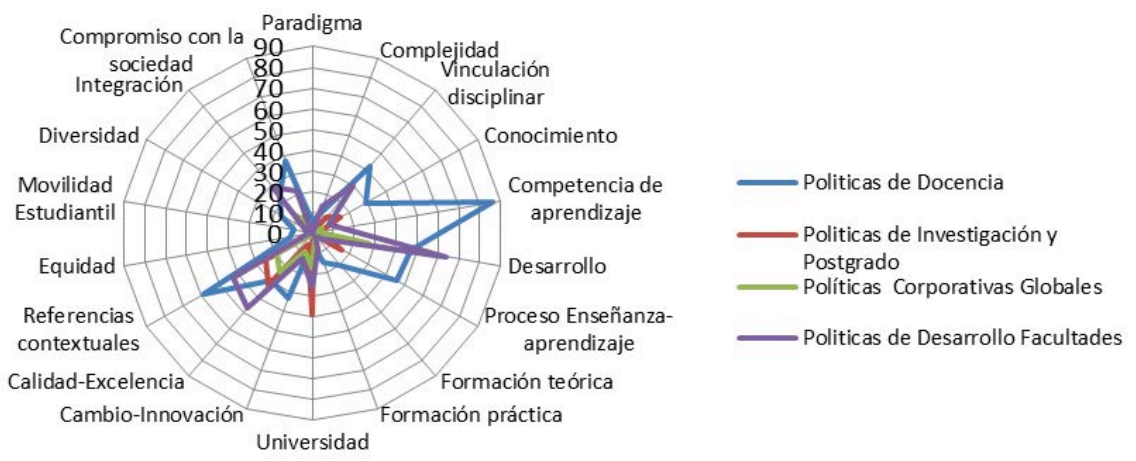

Figura 3. Distribución de frecuencia de codificación Caso 2

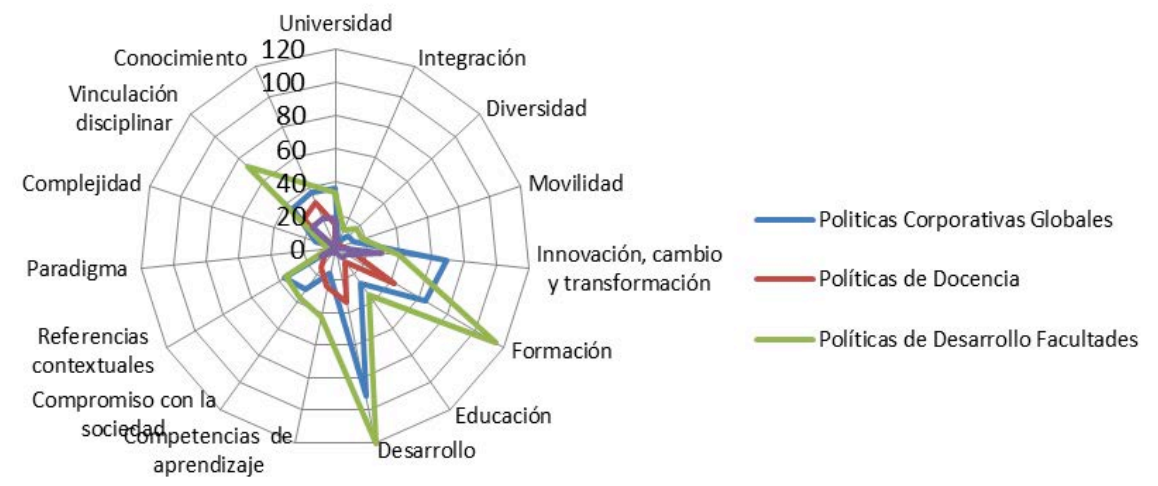


Figura 4. Distribución de frecuencia de codificación Caso 3

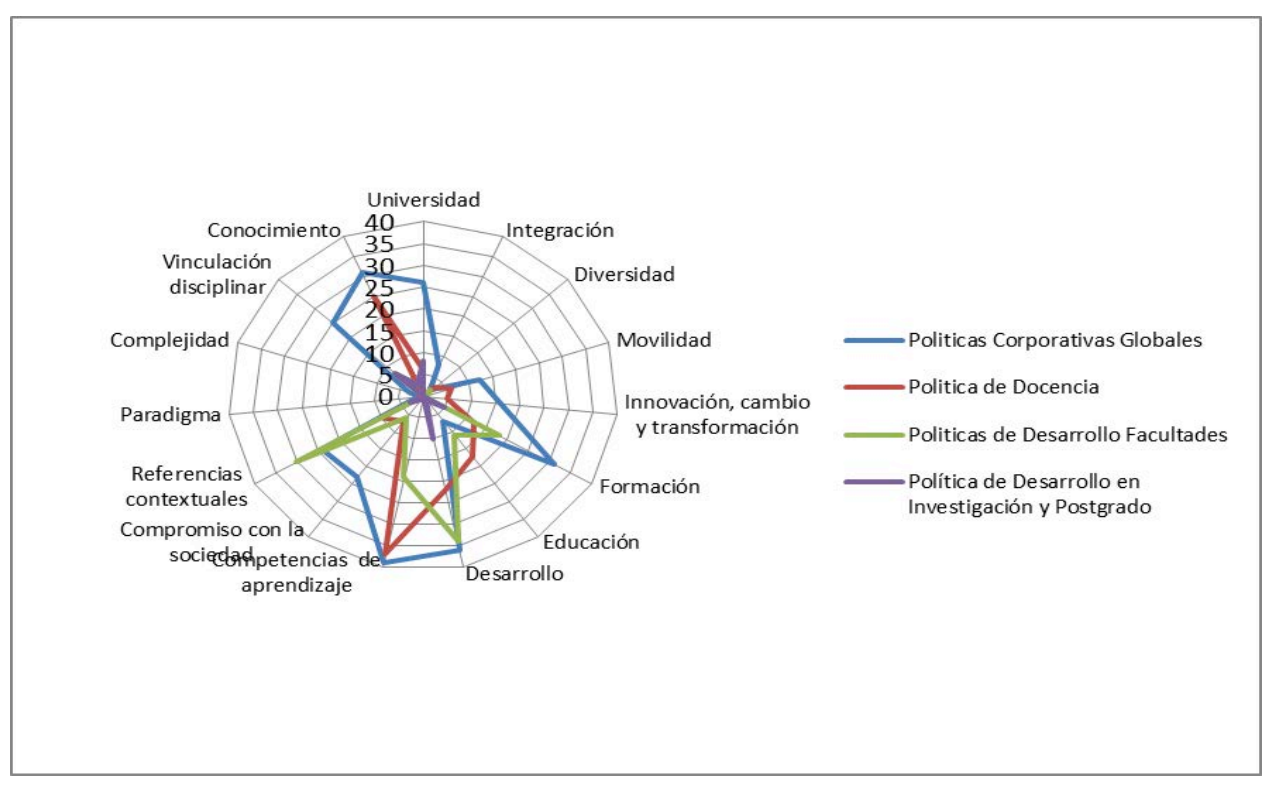

\section{DISCUSIÓN Y CONCLUSIONES}

Considerando el primer caso podemos establecer desde planos generales amplios márgenes interpretativos en los documentos de Políticas de Docencia dentro de la totalidad de códigos referenciados, siendo el código competencias de aprendizaje el que marca una mayor frecuencia dentro de esta tipología. El área de Políticas de Investigación posee una mayor tendencia hacia los códigos como desarrollo, universidad, vinculación disciplinar y referencias al contexto.

El Área de Extensión presenta una tendencia a referencias contextuales y de universidad, haciendo énfasis hacia la identidad de la universidad como organismo vinculado al medio en que se emplaza. El área de Políticas Corporativas Globales presenta bajas tendencias hacia la totalidad de códigos. Es interesante apreciar que este documento de Políticas de Docencia imbrique una diversidad de códigos que permiten la apertura de la universidad hacia una macro-estructura como es la sociedad; referencias contextuales y compromiso con la sociedad nos permiten interpretar que parte del enfoque educativo se amplía hacia contextos extra universitarios. Esta particularidad nos remite a dos operadores propios de la teoría de la complejidad comentados en el Marco teórico-referencial esbozados por Giraldo (2005: 5), el operador dialógico y el operador hologramático. El primero se manifiesta en la creación de lazos comunicantes entre un sistema organizativo y otro, que acostumbran a concebirse como dos formas distintas de desarrollo del ser humano. A la vez, este diálogo permite la imbricación del segundo operador, el cual permite la visualización de un fenómeno incluido de manera particular, con su organización y coherencia interna, en otro fenómeno que es la proyección del primero y que, a la vez, retroactúan de forma simultánea. El código de universidad en gran parte de los 
documentos se aborda de manera autorreferencial, aludiendo a principios, objetivos y fines de la universidad como institución centralizada en la generación de conocimiento. Estas se orientan en la creación de una identidad como organismo educativo intentando poner en primer plano los lineamientos y prospectivas políticas que estas postulan en su organización estratégica y como centro formativo-educativo.

Es interesante observar cómo en el área de Políticas de Investigación se manifiestan los códigos de vinculación disciplinar y referencias contextuales, desde la perspectiva en que estos están asociados interpretativamente dentro de un documento en particular. Dejando aparte su frecuencia y únicamente abocándonos a una coocurrencia hermenéutica en base al marco teórico-referencial, la investigación constituye uno de los principales elementos mediante los cuales se genera conocimiento y plantear esta dentro de espacios de convergencia disciplinaria realizando una serie de referencias hacia al contexto comunitario nos parece un principio lógico que debe emerger desde una postura sistemática-compleja como universidad. Vincular el conocimiento a los contextos particulares significa eclosionar las diversas realidades en las cuales éste conocimiento emerge, más allá de considerar a la universidad como único elemento posibilitador de saberes y competencias. Así mismo podemos imbricar lo anteriormente dicho a los códigos encontrados en el Área de Extensión.

En el caso de programas de carácter más autónomos, se observaron prospectivas destinadas al flujo comunicativo entre disciplinas respaldado por el alto dominio de las concepciones de pluri-multi-inter y transdisciplina, constituyéndose a través de sus lineamientos y proyecciones como un organismo orientado hacia la producción de conocimiento vinculado desde una amplia gama de disciplinas. Sin embargo, al ser un espacio con relativa autonomía dentro de la universidad, la forma de acceder a estos programas, se encuentra regulada por filtros de alto estándar y rendimiento académico.

En los casos 2 y 3 a través del análisis se puede evidenciar que la presencia de nociones de complejidad, paradigma, vinculación disciplinar y conocimiento en las instituciones investigadas, están asociados a códigos de carácter generales; estos aparecen marcados fuertemente por la dominante tendencia hacia una economía de mercado que requiere más bien profesionales que se desempeñen en diversas áreas más que seres humanos que comprendan los fenómenos de asociatividad a partir de los que se construye una sociedad planetaria. En este contexto, la presencia códigos complejos pareciera ser un estándar en donde la utilización de códigos y prácticas complejas está asociado solo ramas de funcionamiento institucional.

Por otra parte, al analizar no solo las frecuencias sino también el contexto que encierra a los códigos complejos en las prospectivas políticas, es posible develar que las características que se le atribuyen a las nociones (códigos) están relacionados con la atribución de denominaciones complejas a espacios físicos que a través de su nombre deberían, aparentemente, contener las implicancias epistémicas de significación del concepto en sí. A pesar de que no es posible encerrar estos conceptos en espacios no cognitivos, es un primer acercamiento dirigido a generar características de complejidad en la universidad.

En el proceso de análisis emerge una multidimensionalidad de factores que pueden influir en la configuración de procesos connotativos que lleven a delimitar los núcleos de sentido en las instituciones participantes de la investigación. De este modo, es posible evidenciar que en ambas universidades existen núcleos de emergencia relacionados con los códigos complejos, asociados con mayor frecuencia a la vinculación disciplinar y al 
conocimiento y estos, a su vez, vinculados a denominaciones de tipo general que tienen relación con códigos generales de innovación y desarrollo, que como se ha mencionado con anterioridad, están fuertemente marcados por las tendencias libremercadistas. Ahora bien, la OCDE establece una serie de parámetros (presentados en el marco teórico) para reconocer las unidades de interés en las que se deben focalizar las instituciones con el fin de cumplir con los estándares de "capital humano" requerido para entrar en el mercado global de la educación.

Dentro del análisis se pueden destacar ciertas convergencias tomando en consideración las diversas dimensiones que constituyen a las universidades como institución formadora y compleja. Encontramos que existe una mayor tendencia a referenciar los códigos complejos en las prospectivas generales o macroinstitucionales en donde los informes de autoevaluación institucional toman principal relevancia. Este fenómeno se atribuye a que los procesos de acreditación de las universidades requieren una mirada interna respecto a las falencias existentes. En este sentido, el incorporar elementos complejos podría evidenciar un mayor interés en vincular las comunidades dentro de ésta.

En cuanto a las divergencias, estas aparecen en una frecuencia mayor en las Políticas de Desarrollo de Facultades, de las cuales se destaca que en la primera universidad analizada cuentan con centros llamados interdisciplinarios, lo que connotaría una mayor apertura respecto a atender las nociones complejas, a diferencia de la segunda institución, en donde estas aparecen a nivel general. Respecto a la particularidad que presentan las divergencias en cuanto a la aparición de códigos, llama la atención el caso de la primera universidad, específicamente en la Facultad de Medicina, en donde está presente con fuerza la evocación a reconocer la importancia de las prácticas interdisciplinarias. Situación que sale de lo común debido a que podría pensarse que estos posicionamientos complejos están más ligados a las áreas humanistas

Si bien las evidencias presentan una alta frecuencia de apariciones de códigos, la naturaleza denotativa de estos propicia posibilidades escasas de ser cualificados como complejos. Si bien a través de un análisis hermenéutico se puede generar una aproximación a las nociones que se construyen en las instituciones, las conclusiones generales que pueden emerger de estas se alejan de la posibilidad de evidenciar elementos con profundidad compleja. Las asociaciones entre los códigos complejos y los generales apuntan hacia las propuestas cada vez más antagónicas en donde la realidad creada cartesianamente se opone terminantemente a la aparición de los procesos naturales, multidimensionales y transversales, es decir, los procesos complejos. No es posible desconocer que se manifiestan diversos dominios de interpretación acerca de la importancia de generar cambios estructurales en los posicionamientos epistemológicos que sustentan la proyección política de la universidad, no obstante, ello comprometerá una reflexión con grados de complejidad creciente de interpretación y la articulación con las bases sociales que sustentan la historia de la construcción civilizatoria, como un diálogo entre los elementos que nos componen.

\section{REFERENCIAS BIBLIOGRÁFICAS}

Aronson, P. (2003). La emergencia de la ciencia transdisciplinar. Revista Cinta de Moebio, n. 18. Beuchot, M. (1997). Tratado de hermenéutica analógica. México: UNAM. 
Browne, R. (2006). Comunicación indisciplinada: iconofagia e iconorrea en los medios de (in) comunicación. Revista Austral de Ciencias Sociales, n. 11, 101-114.

Cambursano, S. (2006). Interdisciplina, transdisciplina y multidisciplina. Prácticas en docencia e investigación. San Fernando: Universidad Nacional de Catamarca.

Cárcamo, H. (2005). Hermenéutica y análisis cualitativo. Revista Cinta de Moebio, n. 23.

Consejo Nacional para la Competitividad. (2009). Hacia una estrategia Nacional de Innovación para la Competitividad. Volumen 1. Santiago de Chile: Gobierno de Chile.

Corral, N. y Bar, A. 2012. Construcción de Racionalidades Disciplinares. Revista Cinta de Moebio, n. $45,215-234$.

Echeverría, R. 1998. Ontología del lenguaje. Santiago: LOM.

Eyzaguirre, N; Marcel, M; Rodríguez, J; Tokman, M. (2005). Hacia la Economía del Conocimiento: El camino para crecer con equidad en el largo plazo. Estudios Públicos, $n$. 97.

Gandarilla, J. (2010). La universidad entrando al siglo XXI por el laberinto de la complejidad. Revista Perfiles educativos, vol. XXXII, n. 127.

Giraldo, G. (2005). Teoría de la complejidad y premisas de legitimidad en las políticas de educación superior. Revista Cinta de Moebio, n. 22.

Max-Neef, M. (2004). Fundamentos de la transdisciplinariedad. Valdivia: Universidad Austral de Chile.

Morin, E. (2001). Introducción al pensamiento complejo. Barcelona: Gedisa.

Morin, E. (2006a). El método. La naturaleza de la naturaleza. Madrid: Cátedra.

Morin, E. (2006b). El método. El conocimiento del conocimiento. Madrid: Cátedra.

Motta, R. (2002). Complejidad, educación y transdisciplinariedad. Revista Polis, vol 1, n. 3.

OECD (2008). Annual Report: Education at a Glance. París: OCDE.

Oliva, I. (2008). Conocimiento, universidad y complejidad: bosquejos epistémicos y metodológicos para una vinculación transdisciplinaria. Revista Estudios Pedagógicos, vol. XXXIV, $n$. 2, $227-243$.

Soler, A. (2010). Conocimiento, complejidad y transdisciplina: Aproximación hermenéutica a las prospectivas de desarrollo universitario en el sur de Chile. Tesis. Valdivia: Magíster en Educación, Universidad Austral de Chile.

Sotolongo, P. y Delgado, C. 2006. La revolución contemporánea del saber y la complejidad social: hacia unas ciencias sociales de nuevo tipo. Buenos Aires : Consejo Latinoamericano de Ciencias Sociales - CLACSO

Toledo, U. (2007). Realidades Múltiples y Mundos Sociales. Revista Cinta Moebio, n. 30, 211-244. 
